\title{
WAVE PROPAGATION DUE TO AN EMBEDDED SEISMIC SOURCE IN A GRADED HALF-PLANE WITH RELIEF PECULIARITIES PART I: MECHANICAL MODEL AND COMPUTATIONAL TECHNIQUE*
}

\author{
I.-K. Fontara, F. Wuttke \\ Institute of Applied Geo-science, \\ Christian-Albrechts-University of Kiel, Germany, \\ e-mails: fontara@gpi.uni-kiel.de, fw@gpi.uni-kiel.de \\ S. PARVANOVA \\ University of Architecture, Civil Engineering and Geodesy \\ Faculty of Structural Engineering, Structural Mechanics, \\ 1, Hr. Smirnenski Blvd, 1046 Sofia, Bulgaria, \\ e-mail: slp_y@abv.bg \\ P. Dineva \\ Institute of Mechanics, Bulgarian Academy of Sciences, \\ Acad. G. Bonchev St., Bl. 4, 1113 Sofia, Bulgaria, \\ e-mail: dineva@gmail.com
}

[Received 10 February 2015. Accepted 09 March 2015]

\begin{abstract}
This work addresses the evaluation of the seismic wave field in a graded half-plane with free-surface and/or sub-surface relief subjected to shear horizontally (SH)-polarized wave, radiating from an embedded seismic source. The considered boundary value problem is transformed into a system of boundary integral equations (BIEs) along the boundaries of the free-surface and of any sub-surface relief, using an analytically derived frequency-dependent Green's function for a quadratically
\end{abstract}

\footnotetext{
${ }^{*}$ Corresponding author e-mail: fontara@gpi.uni-kiel.de

The authors wish to acknowledge support provided through the DFG Grant No. DFG-Wu 496/5-1.

The author P. Dineva acknowledges support by the Bilateral Bulgarian-Greek Projectbased Personnel Exchange Program between BAS and AUTH, Cooperation Program SCIG.

The author S. Parvanova acknowledges support by the DAAD grant code A/14/02829-Ref. 324.
} 
inhomogeneous in depth half-plane. The numerical solution yields synthetic seismic signals at any point of the half-plane in both frequency and time domain following application of Fast Fourier Transform (FFT). Finally, in the companion paper, the verification and numerical simulation studies demonstrate the accuracy and efficiency of the present computational approach. The proposed BIE tool possesses the potential to reveal the sensitivity of the seismic signal to the type and properties of the seismic source, to the existence and type of the material gradient and to the lateral inhomogeneity, due to the free-surface and/or sub-surface relief peculiarities.

KEY WORDS: SH wave, graded half-plane, seismic source, surface relief, site effects, Green's function, BIEM, synthetic seismograms.

\section{Introduction}

Wave propagation through complex geological profiles is of paramount importance in earthquake engineering, due to the ground motion amplifications that may be produced. It is known, that the physical soil and rock properties change from one position to another, focusing on the material inhomogeneity of the geomaterials. The spatial scales over which these properties vary have a direct effect on the seismic wave field. On the other hand, graded geomaterials are challenging regarding modelling and numerical simulation of their inhomogeneous structure and it's strongly influence on the complex seismic field. The mathematical background of models, describing the elastodynamic behaviour of graded materials involves difficulties with the solution of partial differential equations with spatially varying coefficients. That is why in the most of the cases, the discrete models treating inhomogeneity by stratified geological media are used. The discrete models usually are accompanied with the computational techniques as ray theory $[1,2]$, generalized ray technique [3], mode mathing methods $[4,5,6]$, matrix propagation method $[7,8,9]$, reflectivity method [10, 11], wave number integration method [12], discrete wavenumber method [13], finite difference method [14], finite element method [15, 16], boundary integral equation method [17, 18, 19] and hybrid methods [20, 21, 22, 23, 24].

The number of works devoted to dynamically loaded continuously inhomogeneous soil or rock materials is rather limited. The most common used types of inhomogeneous functions, describing the variation of material properties are exponential [25, 26], linear [27], quadratic [28, 29], trigonometric [30]. Manolis and Shaw [31] derived fundamental solutions of wave equation for 2D and 3D inhomogeneous continua, using appropriate algebraic transformations that are valid for certain restricted classes of inhomogeneous materials. Basing on this approach, some results on wave scattering have been produced by cav- 
ities and cracks in continuously inhomogeneous solids [29, 32, 33, 34, 35, 36], using the BIEM. These results were based on the use of closed-form fundamental solutions that were derived by an appropriate functional transformation on the displacement vector, followed by application of the Radon transform.

The presence of different type of heterogeneities as free-surface and subsurface relief peculiarities in inhomogeneous background provokes a new set of difficulties, since wave signals no longer travel undisturbed, but are subjected to amplitude changes and phase angle shifts because of scattering and diffraction phenomena. What can be summarized from the short review in the field is a certain lack of results for seismically-induced ground motion scenarios, when the material properties along the wave path are considered to be smooth functions of the spatial coordinates. Current approaches seem to favour the multi-layered model over the continuously inhomogeneous model, possibly because of inherent difficulties in the latter case that require solving boundary-value problems for differential equations with variable coefficients.

In evaluating the state-of-the-art, we will concentrate mainly on $2 \mathrm{D}$ BIEM based mechanical models, describing continuously inhomogeneous in depth elastic isotropic half-plane with topographic peculiarities under timeharmonic or transient $\mathrm{SH}$-waves radiating from an embedded seismic source. In 1904, Lamb [37] studied the elastic wave produced by the application of the time dependent line load acting normally on the surface of an elastic half-space. Many authors have subsequently viewed and reviewed the problems which deal with the disturbance produced by a point or line source, acting on the surface or buried in an elastic homogeneous half-space with flat free surface. Among them is Pekeris [38], who derived the exact expression for the vertical and horizontal components of the displacement on the surface of a uniform elastic half-space due to a point load with step function time variation, situated on the surface and also at a finite depth. Guan et al. [39] presented a transient Green's function, caused by suddenly applied line load in an isotropic and homogeneous half-space. Antonio and Tadeu [40] presented analytical solutions for computing the 3D displacements in a flat elastic stratum bounded by a rigid base, when it is subjected to spatially sinusoidal harmonic line loads. These functions are also used as Green's functions in a BIEM code that simulates the seismic wave propagation in a confined or semi-confined $2 \mathrm{D}$ valley, avoiding the discretization of the free and rigid horizontal boundaries.

Mainly, two approaches are proposed [41] by BIEM developers for solution of wave propagation problems in half-space and they are based on: (a) the fundamental solution of the governing equation in elastodynamics, see SanchezSesma and Campillo [42, 43] Dineva and Manolis [44, 45] in frequency domain 
and Kamalian et al. [46, 47, 48], Dineva et al. [49] in time domain; (b) the Green's function for half-plane which more over satisfies the boundary condition on the free surface, see Dravinski [50], Ohtsu and Uesugi [51], Ausilio et al. [52], Hirai [53], Belytschko and Chang [54], Panji et al. [55], Wuttke et al. [56]. The BIEM procedure based on the Green's function is mesh-reducing technique, as far as it avoids discretization along the flat free surface, where it satisfies the traction free boundary condition. The development of Green's functions for elastic wave propagation in solids is of engineering importance, because these functions represent fundamental solutions to special types of disturbances (such as the point force and the unit dislocation) and under rather broad boundary conditions (such as the Sommerfeld radiation or free surface condition). The published results in the literature are very limited, when the half-space is continuously inhomogeneous and subjected to waves radiating from a seismic source embedded in the geological media. The most of the papers, concerning wave propagation in a graded half-space consider the seismic load as an incident plane seismic wave, not as wave radiating from a point or line source. Only known to the authors results are in [25], where dynamic response of an inhomogeneous, linear-elastic half-space to a time-harmonic tangential line force on the surface was examined.

To the authors' best knowledge, there are no results concerning the evaluation of the seismic field in a quadratically inhomogeneous half-plane with relief peculiarities and with embedded line seismic source radiating time-harmonic or transient $\mathrm{SH}$-wave.

The aim of this work is to propose an efficient numerical BIEM, based on analytically derived Green's function in Rangelov and Manolis [57] for computation of seismic signals in a quadratically inhomogeneous in depth halfplane with free-surface and sub-surface relief and with an embedded line seismic source radiating time-harmonic or transient SH waves. The goals concern creation of the corresponding software, its verification and its usage for intensive simulations revealing the key role that the material inhomogeneity and heterogeneity plus the type, the properties and the location of the seismic source play on the seismic field.

The paper has following structure: Starting with the problem statement in Section 2, following with the BIEM reformulation of the posed problem in Section 3 and its verification in Section 4. Finally, illustrative numerical examples and accompanied parametric study given in Section 5 serve to illustrate the present methodology and to highlight the differences observed in elastic waves as they propagate through a heterogeneous medium that is no longer homogeneous. Conclusions are discussed in the last Section 6. 


\section{Problem formulation}

In a Cartesian coordinate system $O x_{1} x_{2} x_{3}$ is considered continuously inhomogeneous in depth half-space with free-surface relief in the form of a canyon $V_{\text {can }}$ and subsurface relief as a cavity $V_{\text {cav }}$ of arbitrary shape containing an embedded line seismic source radiating wave of SH-type. The anti-plane deformation state is considered in the plane $x_{3}=0$, where the half-plane $G=R_{-}^{2} \backslash V$ is situated, here $R_{-}^{2}=\left\{x: x=\left(x_{1}, x_{2}\right), x_{2}<0\right\}$ and $V=V_{\text {can }} \cup V_{\text {cav }}$. Denote with $S_{\text {can }}$ the boundary of the canyon, while $S_{2}$ is the part of the free surface line $x_{2}=0$ out of the

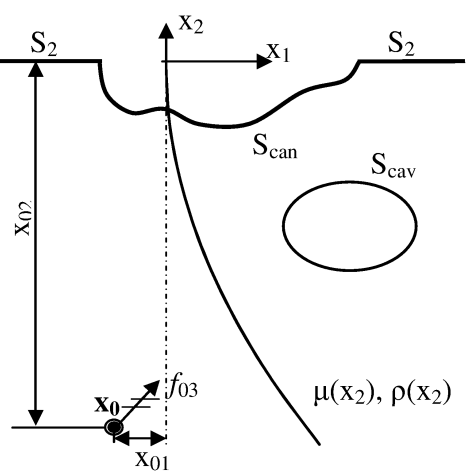

Fig. 1. The problem geometry canyon, so the boundary $\mathrm{S}$ is defined by $S=$ $S_{\text {can }} \bigcup S_{\text {cav }} \bigcup S_{2}$, while the free surface boundary is $S_{\text {freesurface }}=S_{\text {can }} \bigcup S_{2}$. The geometry of the problem is presented in Fig. 1. The seismic load coordinates in the plane $x_{3}=0$ are $\mathbf{x}_{\mathbf{0}}=\left(x_{01}, x_{02}\right)$ and the concentrated seismic body force is $F_{3}\left(\mathbf{x}, \mathbf{x}_{\mathbf{0}}, t\right)=f_{03} g(t) \delta\left(\mathbf{x}, \mathbf{x}_{0}\right)$, where $f_{03}$ and $g(t)$ are its magnitude and time function, respectively. We will consider transient and time harmonic load with frequency $\omega$, where in the latter case $g(t)=e^{i \omega t}$. For the state of anti-plane wave motion, the only non-zero field quantities are displacement component $u_{3}$, stresses $\sigma_{i 3}=\mu\left(x_{2}\right) u_{3, i}, i=1,2$, all depending on the coordinates $\left(x_{1}, x_{2}\right)$ and on time $t$ or frequency $\omega$.

It is considered the case of small deformations and the material is elastic and isotropic. The conservation of linear momentum, i. e. the dynamic equilibrium equation is as follows:

$$
\begin{array}{r}
\sigma_{i 3, i}\left(x_{1}, x_{2}, t\right)=\rho\left(x_{2}\right) \frac{\partial^{2} u_{3}\left(x_{1}, x_{2}, t\right)}{\partial t^{2}}-F_{3}\left(x_{1}, x_{2}, x_{01}, x_{02}, t\right) \\
\text { for transient load } \\
\sigma_{i 3, i}\left(x_{1}, x_{2}, \omega\right)=-\rho\left(x_{2}\right) \omega^{2} u_{3}\left(x_{1}, x_{2}, \omega\right)-F_{3}\left(x_{1}, x_{2}, x_{01}, x_{02}, \omega\right) \\
\text { for time-harmonic load }
\end{array}
$$

here: $\rho\left(x_{2}\right), \mu\left(x_{2}\right)$ are density and shear modulus, comma subscripts denote partial differentiation with respect to the spatial coordinates, while the summation convention over repeated indices is implied. It is assumed that material properties vary with respect to the depth in the following manner:

$$
\mu\left(x_{2}\right)=\mu_{0} h\left(x_{2}\right), \rho\left(x_{2}\right)=\rho_{0} h\left(x_{2}\right),
$$


where $h\left(x_{2}\right)=\left(b x_{2}+1\right)^{2}$ is the inhomogeneity function, $b \leq 0$ is the inhomogeneous coefficient, $\mu_{0}, \rho_{0}$ are the reference constants. Note, that $h\left(x_{2}\right)>0$ for $x \in R_{-}^{2}$.

The boundary value problem for the graded geological media is defined by the partial differential equation Eq. (1) with variable coefficients, depending on the depth and the following boundary conditions:

$$
t_{3}(\mathbf{x})=0 \quad \text { for } \quad\left(x_{1}, x_{2}\right) \in S,
$$

where: $t_{3}=\sigma_{i 3} n_{i}$ is the traction and $\mathbf{n}\left(n_{1}, n_{2}\right)$ is the outward to $G$ normal vector on the boundary S. Additionally the Sommerfeld radiation condition is satisfied at infinity.

The solution of the problem for transient waves is solved by the usage of the following well-known numerical procedure, see Chaillat et al. [58]: (a) direct Fast Fourier Transform (FFT) is applied to the governing equations and the corresponding boundary-value problems in frequency domain are solved by BIEM based on the frequency dependent fundamental solution-Green's function for discrete values of frequencies; (b) inverse FFT is applied to the solutions in frequency domain and finally solutions in time-domain are determined.

\section{BIEM formulation in the frequency domain}

The defined in Section 2 boundary-value problem is solved by BIEM based on the integral representation formula (Dominguez [18] and Manolis and Beskos [17]) and the frequency dependent Green's function for inhomogeneous in depth half-plane. The wave field in inhomogeneous in depth half-plane with an embedded seismic source can be described by boundary integral equation along the boundary of both heterogeneities canyon and cavity $S_{h}=S_{c a n} \cup S_{\text {cav }}$ as follows:

$$
\begin{aligned}
& c u_{3}(\mathbf{x}, \omega)=-\int_{S_{h}} t_{3}^{g^{*}}(\mathbf{x}, \xi, \omega) u_{3}(\xi, \omega) d S_{h} \\
&-\int_{S_{h}} g_{3}^{*}(\mathbf{x}, \xi, \omega) t_{3}(\xi, \omega) d S_{h}+f_{03} \hat{g}(\omega) g_{3}^{*}\left(\mathbf{x}, \mathbf{x}_{0}, \omega\right), \\
& \mathbf{x} \in S_{h}
\end{aligned}
$$

where: $\mathbf{x}$ and $\xi$ are the vector-positions of the source and field points, respectively, $\hat{g}(\omega)$ is the Fourier transform of the time function, c is the jump term depending on the surface geometry at the collocation point, $g_{3}^{*}(\mathbf{x}, \xi, \omega)$ is the frequency-dependent Green's function for quadratically inhomogeneous in depth half-plane and $t_{3}^{g^{*}}(\mathbf{x}, \xi, \omega)$ is its corresponding traction. Once, having 
displacement along the boundary $S_{h}$ we can find displacement at any point in $G$ by the representation formulae:

$$
\begin{aligned}
& u_{3}(\mathbf{x}, \omega)=-\int_{S_{h}} t_{3}^{g^{*}}(\mathbf{x}, \xi, \omega) u_{3}(\xi) d S_{h} \\
&-\int_{S_{h}} g_{3}^{*}(\mathbf{x}, \xi, \omega) t_{3}(\xi) d S_{h}+f_{03} \hat{g}(\omega) g_{3}^{*}\left(\mathbf{x}, \xi_{0}, \omega\right) \\
& \mathbf{x} \in G
\end{aligned}
$$

Green's function $g_{3}^{*}$ is the solution of the following boundary value problem in frequency domain:

$$
\mid \begin{aligned}
& \sigma_{i 3, i}(\mathbf{x}, \omega)+\rho(\mathbf{x}) \omega^{2} g_{3}^{*}(\mathbf{x}, \omega)=-\delta(\mathbf{x}, \xi) \text { for } \mathbf{x} \in R_{-}^{2} ; \xi \in R_{-}^{2} \\
& t_{3}^{g^{*}}(\mathbf{x}, \omega)=\mu(\mathbf{x}) g_{3, i}^{*}(\mathbf{x}, \xi, \omega) n_{i}(\mathbf{x})=0 \text { for } x_{2}=0
\end{aligned},
$$

here: $\left(x_{1}, x_{2}\right)$ is the running point, $\left(\xi_{1}, \xi_{2}\right)$ is the source point, $\delta(\mathbf{x}, \xi)$ is Dirac's delta function. Note, that the derivative in the expression for the traction of the Green's function is with respect to $\left(x_{1}, x_{2}\right)$. In Rangelov and Manolis [57], this Green's function was derived in a closed form by the usage of the smooth transformation of the type $g_{3}^{*}(\mathbf{x}, \xi, \omega)=h^{-1 / 2}(\mathbf{x}) G_{3}^{*}(\mathbf{x}, \xi, \omega)$ and thus, reducing the boundary-value problem with variable coefficients presented by Eqs. (6) to one with constant coefficients with respect to the transformed Green's function $G_{3}^{*}(\mathbf{x}, \xi, \omega)$ available for analytical derivation by the usage of a suitable integral, see details in Rangelov and Manolis [57].

Here, for the completeness of the text, we give the Green's function and the corresponding traction below:

$$
\begin{aligned}
& g_{3}^{*}(\mathbf{x}, \xi, \omega)=h^{-1 / 2}(\xi) h^{-1 / 2}(\mathbf{x}) {\left[\frac{i}{4 \mu_{0}} H_{0}^{(1)}(k r)+\right.} \\
&\left.+\frac{1}{4 \pi \mu_{0}} \int_{-\infty}^{+\infty} \frac{\gamma+b}{\gamma(\gamma-b)} e^{\gamma\left(x_{2}+\xi_{2}\right)} e^{i \eta\left(x_{1}-\xi_{1}\right)} d \eta\right] \\
& t_{3}^{g^{*}}(\mathbf{x}, \xi, \omega)=\mu(\mathbf{x}) g_{3, i}^{*}(\mathbf{x}, \xi, \omega) n_{i}(\mathbf{x})
\end{aligned}
$$


(8) $g_{3,1}^{*}(\mathbf{x}, \xi, \omega)=h^{-1 / 2}(\xi) h^{-1 / 2}(x)\left[\frac{k}{4 \mu_{0}} H_{1}^{(1)}(k r) \frac{\partial r}{\partial x_{1}}+\right.$ $\left.+\frac{1}{4 \pi \mu_{0}} \int_{-\infty}^{+\infty} \frac{\gamma+b}{\gamma(\gamma-b)}(i \eta) e^{\gamma\left(x_{2}+\xi_{2}\right)} e^{i \eta\left(x_{1}-\xi_{1}\right)} d \eta\right]$

$$
\begin{gathered}
g_{3,2}^{*}(\mathbf{x}, \xi, \omega)= \\
=Z_{1}\left[\frac{i}{4 \mu_{0}} H_{0}^{(1)}(k r)+\frac{1}{4 \pi \mu_{0}} \int_{-\infty}^{+\infty} \frac{\gamma+b}{\gamma(\gamma-b)} e^{\gamma\left(x_{2}+\xi_{2}\right)} e^{i \eta\left(x_{1}-\xi_{1}\right)} d \eta\right] \\
+Z_{2}\left[\frac{k}{4 \mu_{0}} H_{1}^{(1)}(k r) \frac{\partial r}{\partial x_{2}}+\frac{1}{4 \pi \mu_{0}} \int_{-\infty}^{+\infty} \frac{\gamma+b}{(\gamma-b)} e^{\gamma\left(x_{2}+\xi_{2}\right)} e^{i \eta\left(x_{1}-\xi_{1}\right)} d \eta\right], \\
Z_{1}=h^{-1 / 2}(\xi)\left(-\frac{1}{2} h^{-3 / 2}(x) \cdot \frac{\partial h}{\partial x_{2}}\right), \\
Z_{2}=h^{-1 / 2}(\xi) h^{-1 / 2}(x),
\end{gathered}
$$

where: $\gamma=\sqrt{\eta^{2}-k^{2}} ; k=k_{1}+i k_{2}, k_{1}^{2}=\frac{\rho_{0}}{\mu_{0}} \omega^{2}, k_{1}^{2}>0 ; k_{2}>0, r=$ $\sqrt{\left(x_{1}-\xi_{1}\right)^{2}+\left(x_{2}-\xi_{2}\right)^{2}}, H_{0}^{(1)}(z)$ and $H_{1}^{(1)}(z)$ is $1^{\text {st }}$ Hankel function of 0 and 1 order, see Gradshteyn and Ryzhik [59]. In the homogeneous case when $b=0$ we obtain the Green's function for homogeneous half-plane for time-harmonic load, see Kobayashi [60]:

$$
g_{3}^{*}(\mathbf{x}, \xi, \omega)=\frac{1}{2 \pi \mu_{0}}\left[K_{0}(-i k r)+K_{0}(-i k \bar{r})\right],
$$

here: $\bar{r}=\sqrt{\left(x_{1}-\xi_{1}\right)^{2}+\left(x_{2}+\xi_{2}\right)^{2}}$.

The authors used this Green's function in Wuttke et al. [56] to study wave propagation in inhomogeneous half-plane with free surface relief, due to the incident plane time harmonic SH-wave. Here, we extend the BIEM approach based on the Green's function to consider the case of graded half-plane with an embedded seismic source and also with sub-surface /free-surface relief. To the best authors' knowledge, there is no solution of this problem in the literature. 


\section{REFERENCES}

[1] Babich, V. M. Ray Method for the Computation of the Intensity of Wavefronts, Moscow, Nauka, 1956, (in Russian).

[2] Chapman, C. H. A New Method for Computing Synthetic Seismograms. Geophysical Journal of the Royal Astronomical Society, 54 (1978), 481-518.

[3] PaO, Y. H., R. G. Gajewski. The Generalized Ray Theory and Transient Response of Layered Elastic Solids. Physical Acoustics (Eds Mason, W. P., R. N. Thurston), New York, Academic Press, 13 (1977), 183-265.

[4] Panza, G. F. Synthetic Seismograms: The Rayleigh Waves Modal Summation. Journal of Geophysics, 58 (1985), 125-145.

[5] Panza, G. F. Synthetic Seismograms from Multimode Summation-Theory and Computational Aspects. Acta Geod. Geph. Hung., 28 (1993), No. 1-2, 197-247.

[6] Panza, G. F., F. Romanelli, Fr. Vaccari. Seismic Wave Propagation in Laterally Heterogeneous Anelastic Media: Theory and Applications to Seismic Zonation. Advances in Geophysics, 43 (2000), 1-95.

[7] Thomson, W. T. Transmission of Elastic Waves through a Stratified Solid. Journal of Applied Physics, 21 (1950), 89-93.

[8] Haskell, N. A. The Dispersion of Surface Waves on Multilayered Media. Bulletin Seismological Society of America, 43 (1953), 17-34.

[9] Knopoff, L. A Matrix Method of Elastic Wave Problems. Bulletin Seismological Society of America, 54 (1964), 431-438.

[10] Fuchs, K., G. MÜLLER. Computation of Synthetic Seismograms with the Reflectivity Method and Comparison with Observations. Geophys. J. R. Astr. Soc., 23 (1971), 417-433.

[11] Kind, R. The Reflectivity Method for a Buried Source. J. Geophys. Res., 44 (1978), 603-612.

[12] Wuttke, F. Beitrag zur Standortidentifzierung mit Oberfachenwellen, PhD thesis, Germany, Weimar, Bauhaus Universitat, 2005.

[13] Bouchon, M. A Simple Method to Calculate Green's Functions for Elastic Layered Media. Bull. Seism. Soc. Am., 71 (1981), 959-971.

[14] Moczo, P. Finite-difference Technique for SH Waves in 2-D Media using Irregular Grids: Application to the Seismic Response Problem. Geophys.J. Int., 99 (1989), 321-329.

[15] Gatmiri, B., C. Arson, K. V. NguYen. Seismic Site Effects by an Optimized 2D BE/FE Method. I. Theory, Numerical Optimization and Application to Topographical Irregularities. Soil Dynamics and Earthquake Engineering, 28 (2008), No. 8, 632-645.

[16] Gatmiri, B., C. Arson. Seismic Site Effects by an Optimized 2D BE/FE Method. II. Quantification of Site Effects in Two-dimensional Sedimentary Valleys. Soil Dynamics and Earthquake Engineering, 28 (2008), No. 8, 646-661.

[17] Manolis, G. D., D. E. Beskos. Boundary Element Methods in Elastodynamics, London, Unwin and Allen, 1987. 
[18] Dominguez, J. Boundary Elements in Dynamics, Southampton, Computational Mechanics Publications, 1993.

[19] Bouchon, M., F. J. SÁnchez-Sesma. Boundary Integral Equations and Boundary Elements Methods in Elastodynamics. Adv. Geophys., 48 (2007), 157189.

[20] Zahradnik, J., P. Moczo. Hybrid Seismic Modelling Based on Discrete Wave Number and Finite Difference Methods. PAGEOPH, 148 (1996), No. 1-2, 21-38.

[21] Moczo, P., E. Bystricky, J. Kristek, M. Carcione, M. Bouchon. Hybrid Modelling of P-SV Seismic Motion at Inhomogeneous Viscoelastic Topographic Structures. B. Seismol. Soc. Am., 87 (1997), No. 5, 1305-1323.

[22] Wuttke, Fr., P. Dineva, T. Schanz. Seismic Wave Propagation in Laterally Inhomogeneous Geological Region via a New Hybrid Approach. Journal of Sound and Vibration, 330 (2011), 664-684.

[23] Panza, G., I. Paskaleva, P. Dineva, Cr. La Mura. Earthquake Site Effects Modelling by Hybrid MS-BIEM: The Case Study of Sofia Bulgaria. Rendiconti di Scienze Fisiche by the Accademia dei Lincei, 20 (2009), 91-116.

[24] Manolis, G. D., K. Makra, P. Dineva, T. Rangelov. Seismic Motions in a Non-homogeneous Soil Deposit with Tunnels by a Hybrid Computational Technique. Earthquakes and Structures, 5 (2013), No. 2, 161-205.

[25] Vrettos, Сн. Dispersive SH-surface Waves in Soil Deposits of Variable Shear Modulus. Soil Dyn. Earthq. Eng., 9 (1990), 255-264.

[26] Vretros, Сн. Forced Anti-plane Vibrations at the Surface of an Inhomogeneous Half-space. Soil Dynamics and Earthquake Engineering, 10 (1991), No. 5, 230-235.

[27] Vardoulakis, I., C. Vrettos. Dispersion Law of Rayleigh-type Waves in a Compressible Gibson Half-space. Int. J.Numer. Anal. Meth. Geomech., 12 (1988), 639-655.

[28] Manolis, G. D., T. V. Rangelov, R. P. Shaw. Conformal Mapping Methods for Variable Parameter Elastodynamics. Wave Motion, 36 (2002), 185-202.

[29] Manolis, G. D., P. S. Dineva, T. V. Rangelov. Wave Scattering by Cracks in Inhomogeneous Continua using BIEM. Int. J. Solids Str., 41 (2004), 3905-3927.

[30] KaKar, R., S. KaKar. Propagation of Love Waves in a Non-homogeneous Elastic Media. J. Acad. Indus. Res., 1 (2012), No. 6, 323-328.

[31] Manolis, G. D., R. P. Shaw. Green's Function for the Vector Wave Equation in a Mildly Heterogeneous Continuum. Wave Motion, 24 (1996), 59-83.

[32] Manolis, G. D., T. V. Rangelov, P. Dineva. Free-field Wave Solutions in a Half-plane Exhibiting a Special-type of Continuous Inhomogeneity. Wave Motion, 44 (2007), 304-321.

[33] Manolis, G. D., T. V. Rangelov, P. Dineva. Free-field Dynamic Response of an Inhomogeneous Half-space. Arch. Appl. Mech., 79 (2009), 595-603.

[34] Manolis, G. D., P. Dineva, T. V. Rangelov. Dynamic Fracture Analysis of a Smoothly Inhomogeneous Plane Containing Defects by BEM. Eng. Anal. Bound. Elem., 36 (2012), 727-737. 
[35] Dineva, P., G. D. Manolis, T. V. Rangelov. Sub-surface Crack in Inhomogeneous Half-plane: Wave Scattering Phenomena by BEM. Eng. Anal. Bound. Elem., 30 (2006), No. 5, 350-362.

[36] Dineva, P., T. V. Rangelov, G. D. Manolis. Elastic Wave Propagation in a Class of Cracked Functionally Graded Materials by BEM. Computat. Mech., 39 (2007), No. 3, 293-308.

[37] Lamb, H. On the Propagation of Tremors over the Surface of an Elastic Solid, Phil. Trans. R. Soc., London, Series A203, 1904.

[38] Pekeris, C. L. The Seismic Buried Pulse. Proc. Nat. Acad. Sci., 41 (1955), 629-639.

[39] Guan, F., I. D. Moore, C. C. Spyrakos. Two Dimensional Transient Fundamental Solution due to Suddenly Applied Load in a Half-space. Soil Dyn. Earthquake Eng., 17 (1998), No. 4, 269-277.

[40] Antonio, J., A. TAdeu. 3D Seismic Response of a Limited Valley via BEM using 2.5D Analytical Green's Functions for an Infinite Free-rigid Layer. Soil Dynamics and Earthquake Engineering, 22 (2002), 659-673.

[41] Dominguez, J., T. Meise. On the use of the BEM for Wave Propagation in Infinite Domains. Eng. Anal. Bound. Elem., 8 (1991), No. 3, 132-138.

[42] Sanchez-Sesma, F. J., M. Campillo. Diffraction of P, SV, and Rayleigh Waves by Topographic Features: a Boundary Integral Formulation. Bull. Seism. Soc. Am., 81 (1991), No. 6, 2234-2253.

[43] Sanchez-Sesma, F. J., M. Campillo. Topographic Effects for Incident P, SV and Rayleigh Waves. Tectonophysics, 218 (1993), 113-125.

[44] Dineva, P., G. Manolis. Scattering of Seismic Waves by Cracks in Multilayered Geological Regions: I. Mechanical Model. Soil Dynamics and Earthquake Engineering, 21 (2001a), 615-625.

[45] Dineva, P., G. Manolis. Scattering of Seismic Waves by Cracks in Multilayered Geological Regions: II. Numerical Results. Soil Dynamics and Earthquake Engineering, 21 (2001b), 627-641.

[46] Kamalian, M., M. K. Jafari, A. Sohrabi-Bidar, A. Razmkhah, B. GatMIRI. Time-domain Two-dimensional Site Response Analysis of Nonhomogeneous Topographic Structures by a Hybrid FE/BE Method. Soil Dyn. Earthq. Eng., 26 (2006), No. 8, 753-765.

[47] Kamalian, M., B. Gatmiri, A. Sohrabi-Bidar, A. Khalaj. Amplification Pattern of 2D Semi-sine Shaped Valleys Subjected to Vertically Propagating Incident Waves. Commun. Numer. Methods Eng., 23 (2007), No. 10, 871-887.

[48] Kamalian, M., M. K. Jafari, A. Sohrabi-Bidar, A. Razmkhah. Seismic Response of 2D Semi-sines Shaped Hills to Vertically Propagating Incident Waves: Amplification Patterns and Engineering Applications. Earthq. Spectra, 24 (2008), No. 2, 405-430.

[49] Dineva, P., P. Borejko, L. Hadjikov, F. Zigler. Transient Elastic Waves in a Half-space: Comparison of the DBIE - Method with the Method of Generalized Ray. Acta Mechanica, 115 (1996), 203-211. 
[50] Dravinski, M. Influence of Interface Depth upon Strong Ground Motion. Bull. Seism. Soc. Am., 72 (1982), No. 2, 597-614.

[51] Ohtsu, M., S. Uesugr. Analysis of SH Wave Scattering in a Half Space and its Applications to Seismic Responses of Geological Structures. Eng. Anal., 2 (1985), No. 4, 198-204.

[52] Ausilio, E., E. Conte, G. Dente. Seismic Response of Alluvial Valleys to SH Waves. Seism. Eng. Conf., AIP Conf. Proc., 1020 (2008), 199-206.

[53] Hirai, H. Analysis of Transient Response of SH-wave Scattering in a Half-space by the Boundary Element Method. Eng. Anal., 5 (1988), No. 4, 189-194.

[54] Belytschko, T., H. S. Chang. Simplified Direct Time Integration Boundary Element Method. J. Eng. Mech., 114 (1988), No. 1, 117-134.

[55] Panji, M., M. Kamalian, J. A. Marnani, M. K. Jafari. Transient Analysis of Wave Propagation Problems by Half-plane BEM. Geophysical Journal International, 194 (2013), 1849-1865.

[56] Wuttke, F. R., I.-K. Fontara, P. Dineva, Ts. Rangelov. SH-wave Propagation in a Continuously Inhomogeneous Half-plane with Free Surface Relief by BIEM. ZAMM, DOI 10.1002/zamm.201300198, (2014).

[57] Rangelov, T., G. Manolis. Point Force and Dipole Solutions in the Inhomogeneous Half-plane under Time-harmonic Conditions. Mech. Res. Commun., 56 (2014), 90-97.

[58] Chaillat, S., M. Bonnet, J. F. Semblat. A New Fast Multi-domain BeM to Model Seismic Wave Propagation and Amplification in 3-D Geological Structures. Geophys. J. Int., 177 (2009), 509-531.

[59] Gradshteyn, I. S., I. M. Ryzhik. Table of Integrals, Series and Products, New York, Academic Press, 1980.

[60] Kobayashi, S. Some Problems of the Boundary Integral Equation Method in Elastodynamics. In: C. A. Brebbia, T. Futagami, and M. Tanaka, editors, Boundary Elements V, Berlin, Springer-Verlag, 1983, 775-784,

[61] Matlab, The language of Technical Computing, Version 7.7. The Math-Works Inc., Massachusetts, Natick, 2008.

[62] Achenbach, J. D. Wave Propagation in Elastic Solids, North Holland, Amsterdam, 1973.

[63] Yeh, C. S., T. J. Teng, W. I. Liao. On Evaluation of Lamb's Integrals for Waves in a Two-domain Elastic Half-space. The Chinese Journal of Mechanics, 16 (2000), No. 2, 109-124.

[64] Shah, A. H., K. C. Wong, S. K. Datta. Diffraction of SH Waves in a Halfspace. Earthq. Eng. Struct. Dyn., 10 (1982), 519-528. 